\title{
Increased diagnostic yield by reanalysis of data from a hearing loss gene panel
}

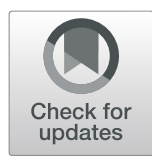

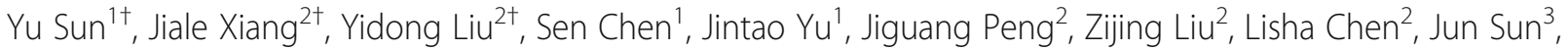
Yun Yang ${ }^{2}$, Yaping Yang ${ }^{4,5}$, Yulin Zhou ${ }^{6,7^{*}}$ and Zhiyu Peng ${ }^{2^{*}}$ (D)

\begin{abstract}
Background: Congenital hearing loss affects approximately 1-2 infants out of every 1000, with 50\% of the cases resulting from genetic factors. Targeted gene panels have been widely used for genetic diagnosis of hearing loss. This study aims to reveal new diagnoses via reanalyzing historical data of a multigene panel, and exam the reasons for new diagnoses.

Methods: A total of 210 samples were enlisted, including clinical reports and sequencing data of patients with congenital/prelingual hearing loss who were referred to clinical genetic testing from October 2014 to June 2017. All variants listed on the original clinical reports were reinterpreted according to the standards and guidelines recommended by the American College of Medical Genetics and Genomics and the Association for Molecular Pathology (ACMG/AMP). Expanded analysis of raw data were performed in undiagnosed cases.

Results: Re-analysis resulted in nine new diagnoses, improving the overall diagnostic rate from 39 to $43 \%$. New diagnoses were attributed to newly published clinical evidence in the literature, adoption of new interpretation guidelines and expanded analysis range.

Conclusion: This work demonstrates benefits of reanalysis of targeted gene panel data, indicating that periodical reanalysis should be performed in clinical practice.
\end{abstract}

Keywords: Reanalysis, Variant interpretation, Multigene panel, Hearing loss, Diagnostic yield

\section{Background}

Congenital hearing loss affects approximately 1-2 infants out of every 1000 , with $50 \%$ of the cases resulting from genetic factors [1]. Molecular diagnosis of hearing loss can help direct the genetic counseling and clinical management of probands and their family members [2]. One of the molecular testing methods used to diagnose hearing loss is Sanger sequencing. However, Sanger sequencing diagnoses usually begin by testing a limited number of selected genes (generally starting with GJB2 in hearing loss), resulting in a low detection rate [3]; well-established genetic knowledge about the target

\footnotetext{
*Correspondence: zhou_yulin@126.com; pengzhiyu@bgi.com

†Yu Sun, Jiale Xiang and Yidong Liu contributed equally to this work.

GUnited Diagnostic and Research Center for Clinical Genetics, School of

Public Health of Xiamen University, Xiamen, Fujian 361003, China

${ }^{2}$ BGI Genomics, BGI-Shenzhen, Shenzhen 518083, China

Full list of author information is available at the end of the article
}

population may therefore be necessary to determine which genes should be tested first. Furthermore, the genetic heterogeneity of hereditary hearing loss [4] makes sequential gene-by-gene testing unrealistic and costly.

With the advance of next generation sequencing (NGS) techniques, targeted genomic capture and massively parallel sequencing has become an important diagnostic tool for hereditary hearing loss [5]. This method can be used to examine over one hundred known deafness-related genes simultaneously. The diagnostic yield of comprehensive NGS hearing loss testing panels is close to $40 \%[4,6]$, much higher than that of Sanger sequencing.

It was recently reported that reanalysis of genome-wide NGS data with updated knowledge can improve the diagnostic rate $[7,8]$. For instance, diagnostic yield increased by $13 \%$ following reanalysis of genome-wide data from patients with severe developmental disorders [9] and by $11 \%$

(C) The Author(s). 2019 Open Access This article is distributed under the terms of the Creative Commons Attribution 4.0 International License (http://creativecommons.org/licenses/by/4.0/), which permits unrestricted use, distribution, and 
in a cohort of 37 families with suspected Mendelian disorders (primarily intellectual disabilities) [10]. Reanalysis of data from gene panels targeting known disease genes will not yield new diagnoses resulting from novel gene discovery since the panels have a fixed design, but it may still be beneficial due to improvements in variant interpretation. However, reports of such reanalysis are currently lacking.

In this study, we recruited patients with congenital/ prelingual hearing loss. We sought to reveal new diagnoses via reanalyzing their targeted gene panel data, and exam the reasons for new diagnoses.

\section{Methods}

\section{Patients}

A total of 210 patients with congenital or prelingual hearing loss, defined as detection before three years of age, were retrospectively studied. They had been referred to clinical genetic testing from October 2014 to June 2017 and consented to anonymous use of their data for scientific research. Healthy relatives of the patients were not included. The Institutional Review Board of the BGI approved this study.

\section{HearingCare NGS testing}

After a clinical diagnosis of hearing loss, peripheral blood samples were submitted for testing with commercial exome sequencing panels, either HearingCare_127 or HearingCare_81. The two tests use the same gene panel but involve analysis of a different number of genes, with 127 genes analyzed in HearingCare_127 and 81 in HearingCare_81. The gene list is presented in Additional file 1: Table S1. All tests were performed using target capture (Agilent, Santa Clara, CA, USA) followed by sequencing on a Hiseq-2500 (Illumina, San Diego, CA, USA). The coding regions and splice sites $( \pm 10 \mathrm{bp})$ of the target genes were analyzed. Bioinformatics pipelines included alignment of sequencing reads using the Burrows-Wheeler Aligner (0.7.12) [11] and variant calling using the Genome Analysis Tool Kit (GATK 3.4) [12].

\section{Variant filtering and prioritization}

Variant filtering and prioritization were first based on population databases (the Exome Aggregation Consortium (ExAC), the Genome Aggregation Database (gno$\mathrm{mAD}$ ), Exome Sequencing Project v. 6500 (ESP6500), 1000 Genomes and local databases). Variants at a minor allele frequency of $>1 \%$ in either one of the databases were excluded except for hotspot variants, such as NM_004004.5(GJB2):c.109G > A. Then, the functional consequences of the remaining variants were predicted by Condel [13]. If no diagnosis was found for a patient through single nucleotide variants (SNVs), then copy number variants $(\mathrm{CNV})$ were characterized. An in-house spreadsheet that computes the inter-sample normalized depth of coverage per exon was used starting in 2015. All reported SNVs were confirmed via Sanger sequencing and CNVs were confirmed via qPCR.

\section{Variant interpretation and reporting}

Variants were interpreted according to the standards and guidelines published in the literature [14-16]. Each variant was classified into one of five categories: pathogenic (P), likely pathogenic (LP), variant of uncertain significance (VUS), likely benign (LB) or benign (B).

In clinical reports, P/LP variants were all listed. However, VUS in undiagnosed patients were only reported if certain conditions were met. If a VUS was found concurrently in a gene with a P/LP variant, the VUS was reported. In cases where no P/LP variant was curated, a VUS was reported based on patients' phenotypes.

\section{Reanalysis workflow}

The reanalysis workflow consisted of reinterpretation and expanded analysis (Fig. 1). The purpose of variant reinterpretation was to reassess the pathogenicity of variants (Table 1). All variants were reinterpreted according to the standards and guidelines recommended by the American College of Medical Genetics and Genomics and the Association for Molecular Pathology (ACMG/ AMP) [17]. Undiagnosed cases were then processed through expanded analysis, which focused on CNV detection and examined 127 genes irrespective of the initially tested panel (Table 1). Those remained undiagnosed should be subject to periodic reanalysis.

\section{Statistical analysis}

For categorical data, summary data were reported as frequencies and percentages, and chi-square tests were used for between-group comparisons. A $P$ value of less than 0.05 was considered statistically significant. Statistical analysis was performed with IBM SPSS Statistics, version 24 (SPSS).

\section{Results}

\section{Cohort and overall results}

Out of 210 patients, $49 \%$ were male. A total of $52 \mathrm{pa}$ tients $(25 \%)$ have a self-reported family history of hearing loss. The majority of cases had been tested with HearingCare_127 (83\%; 174), while the remainder used HearingCare_81 (17\%; 36) (Table 2).

Molecular diagnoses were initially made in 82 out of 210 patients (39\%). The diagnostic yields of HearingCare_127 and HearingCare_81 were 41 and 28\%, respectively $(p=0.128)$. The diagnostic rate was not significantly different in patients with or without a family history $(40 \%$ vs. $39 \%, p=0.820)$ or in patients from different calendar years $(p=0.220)$. In diagnosed patients, 


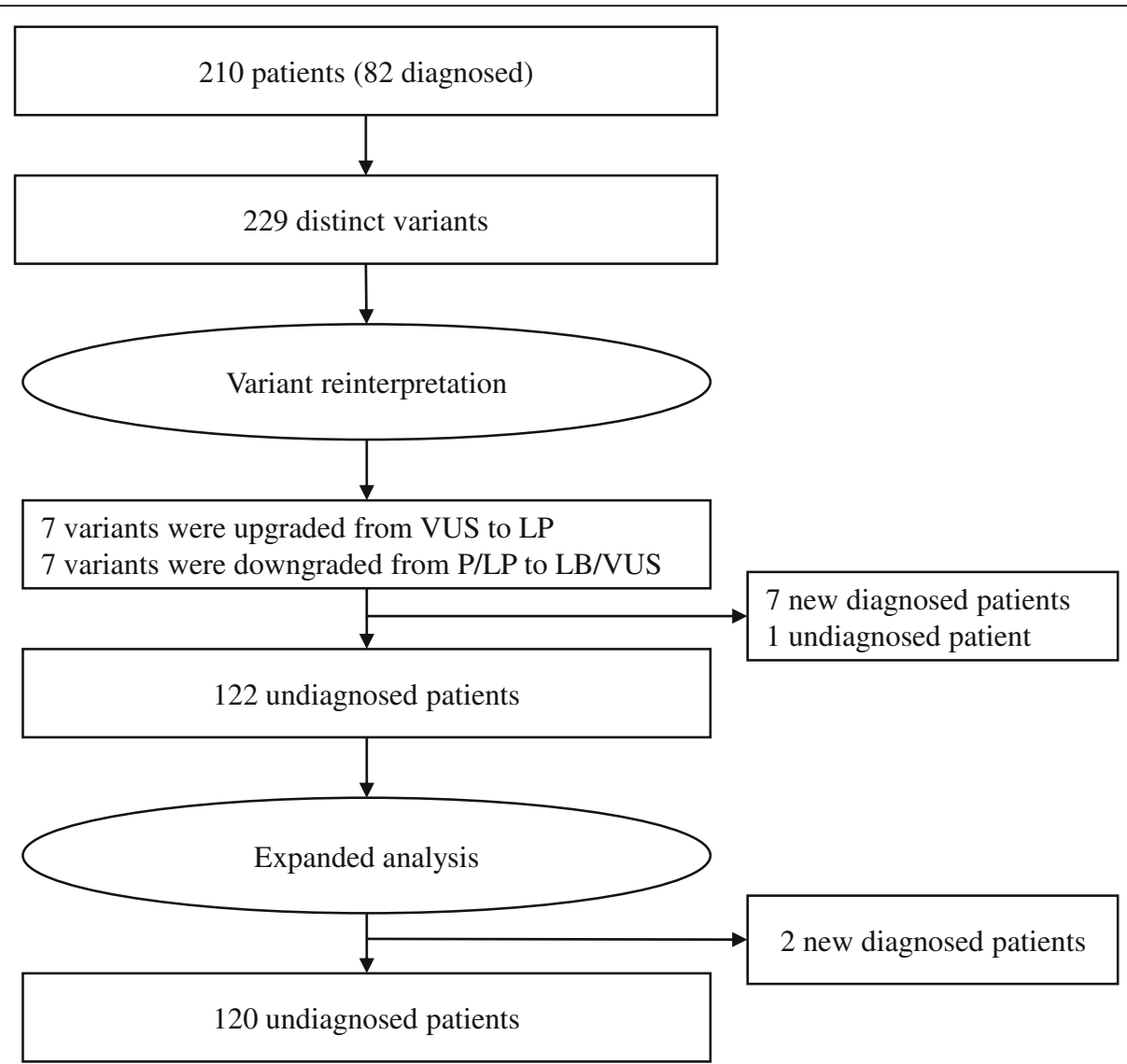

Fig. 1 Flowchart of variant reinterpretation and expanded analysis. P, pathogenic; LP, likely pathogenic; VUS, variant of uncertain significance; $L B$, likely benign. The one undiagnosed patient was attributed to the downgraded of a X-linked dominant variant (NM_000495.4(COL4A5):C.2858G > T) from likely pathogenic to likely benign

GJB2 and SLC26A4 were the most significant contributors, present in 45 and $35 \%$ of cases, respectively. As expected, autosomal recessive inheritance was the most common inheritance pattern in congenital/prelingual hearing loss patients (Additional file 2: Table S2).

\section{Improvement of diagnosis}

The overall diagnostic rate improved from 39 to $43 \%$ (Table 2). Of the nine new diagnoses, five patients ( $\mathrm{Pa}-$ tients 1-5) were upgraded in light of new evidence from studies published after the reports were first released. Pathogenic moderate evidence (PM5: a different missense change determined to be pathogenic has been seen before) from ACMG/AMP guidelines [17] was applied twice to upgrade two reports (Patient 6 and 7). In addition, a pathogenic variant with autosomal dominant inheritance was discovered in a gene which was beyond the initial analysis range (Patient 8). A CNV was found in patient 9 via expanded analysis, paired as a compound heterozygote with a single-nucleotide variant (Table 3 ). All the new diagnoses were attributed to the recategorization of a single variant; none were due to the recategorization of two different AR variants in the same gene. The new diagnoses were not enriched in a specific year.

In addition, one initial diagnosed case was reclassified to undiagnosed due to downgrade of a X-linked

Table 1 Purpose and potential sources of improvement by variant reinterpretation and expanded analysis

\begin{tabular}{lll}
\hline Step & Purpose & Potential sources of improvement \\
\hline Variant reinterpretation & To reassess the pathogenicity of variants & $\begin{array}{l}\text { New evidence from publications to upgrade the } \\
\text { pathogenicity }\end{array}$ \\
& $\begin{array}{l}\text { New standards and guidelines for variant interpretation } \\
\text { Expanded analysis }\end{array}$ & $\begin{array}{l}\text { Missed copy number variants } \\
\text { To detect copy number variants } \\
\text { To expand analysis to other hearing } \\
\text { loss related genes }\end{array}$ \\
\hline
\end{tabular}


Table 2 Characteristics and diagnosed yield of the study cohort

\begin{tabular}{|c|c|c|c|}
\hline & $\begin{array}{l}\text { Patients, } \\
\text { No. (\%) }\end{array}$ & $\begin{array}{l}\text { Diagnosed yield } \\
\text { before reanalysis, } \\
\text { No. (\%) }\end{array}$ & $\begin{array}{l}\text { Diagnosed yield } \\
\text { after reanalysis, } \\
\text { No. (\%) }\end{array}$ \\
\hline All & $210(100)$ & $82(39)$ & $90(43)$ \\
\hline \multicolumn{4}{|l|}{ Sex } \\
\hline Male & $102(49)$ & $42(41)$ & $44(43)$ \\
\hline Female & $108(51)$ & $40(37)$ & $46(43)$ \\
\hline \multicolumn{4}{|l|}{ Family history } \\
\hline Yes & $52(25)$ & $21(40)$ & $25(48)$ \\
\hline No & $158(75)$ & $61(39)$ & $65(41)$ \\
\hline \multicolumn{4}{|l|}{ Selection } \\
\hline HearingCare_127 & $174(83)$ & $72(41)$ & $77(44)$ \\
\hline HearingCare_81 & $36(17)$ & $10(28)$ & $13(36)$ \\
\hline \multicolumn{4}{|l|}{ Year } \\
\hline 2014 & $10(5)$ & $2(20)$ & $4(40)$ \\
\hline 2015 & $62(30)$ & $30(48)$ & $32(52)$ \\
\hline 2016 & $93(44)$ & $33(35)$ & $35(38)$ \\
\hline 2017 & $45(21)$ & $17(38)$ & $19(42)$ \\
\hline
\end{tabular}

dominant variant (NM_000495.4(COL4A5):c.2858G > T) from likely pathogenic to likely benign (Fig. 1). The variant was interpreted and reported in March 2015. The availability of public genomic databases, such as ExAC and gnomAD [18], provided population evidence to reclassify this variant to likely benign. Moreover, we noted that the 7 upgraded variants resulted in 7 new diagnoses (7/7; 100\%), whereas 7 downgraded variants only resulted in 1 patient from "diagnosed" to "undiagnosed" $(1 / 7 ; 14 \%)$, reaching a significant difference $(P<0.001)$.

\section{New publications (patients 1-5)}

Variants that were reclassified from VUS to likely pathogenic in patients 1-5. The upgrade of NM_016 239.3(MYO15A):c.10245_10247delCTC resulted in two probands getting diagnosed (patient 1 and 2). This variant was first reported in 2015 as a compound heterozygote with MYO15A c.8198A >C [19]. Because the pathogenicity of the latter variant was undetermined, the pathogenicity of c.10245_10247delCTC could not be upgraded. This changed in February 2018 when a new publication provided solid evidence that the variant segregated with nonsyndromic hearing loss in a Korean family [20]. The pathogenicity of the variant in patient 3 was also supported by evidence of segregation [21].

In patients 4 and 5, two missense amino acid changes occurred in MITF at the same position. The pathogenicity of p.Arg341Cys was reclassified because it was proven to be de novo and to cause prelingual hearing loss in a five-year-old girl in April 2016 [22]. The reclassification of p.Arg341Cys in turn supported the pathogenicity of Arg341Gly. Two years later, p.Arg341Gly was reported as a pathogenic variant in a Chinese family in June 2018 [23], and in a Indian family in November 2018 [24], further supporting its pathogenicity. It is worth noting that NM_198159.2(MITF):c.1021C > T (p.Arg341Cys) was discovered from original file of an unreported VUS in patient 5, who presented only congenital profound hearing loss when they were referred for genetic testing.

\section{ACMG/AMP guidelines (patients 6-7)}

Two patients were reclassified due to the application of standards and guidelines for the interpretation of sequence variants recommend by ACMG/AMP [17]. Specifically, TMC1 p.Gly417Glu and $C D H 23$ p.Pro346Arg were detected in patient 6 and patient 7 , respectively. Pathogenic missense changes at the positions of these two variants have been established before $[25,26]$, providing moderate evidence to reclassify the variants.

\section{Expanded analysis (patients 8-9)}

Patient 8 was clinically diagnosed with nonsyndromic hearing loss, and their sample was tested using HearingCare_81 panel. The number of genes analyzed was increased to 127 during reanalysis. An autosomal dominant frameshift variant, NM_001127366.2(PAX3):c.870_871insC, was discovered and is known to be the genetic cause of Waardenburg Syndrome [27]. Penetrance of individuals with Waardenburg Syndrome varied, and sensorineural hearing loss was a presenting feature in $47 \% \sim 58 \%$ patients [27].

Patient 9 was reported to have congenital hearing loss without a family history, and his report listed only the pathogenic variant c.1333C $>\mathrm{T}$ in $T M C 1$ alone at December 2014. The expanded analysis uncovered a CNV in conjunction with this SNV. The exon-level deletion from exon 6 to exon 10 was missed in the earlier analysis because $\mathrm{CNV}$ analysis was not available at that time.

\section{Discussion}

In this study, we increased the diagnostic yield of congenital/prelingual hearing loss patients from 39 to $43 \%$ by reanalysis of targeted gene panel data. Considering that the contribution of genetic factors to congenital hearing loss is around 50\% [1], the improvement to $43 \%$ is significant. The residual cases might be attributable to untargeted, novel, or unknown deafness-related genes, non-exonic sequence variants, or structural variations that could be detected by whole genome/exome sequencing $[9,28,29]$.

Reanalysis of targeted gene panel data for a specific disease is reliable and valuable. To date, all published reanalysis research has focused on WES data for a wide spectrum of disorders $[7,9,10]$. The diagnostic yield 


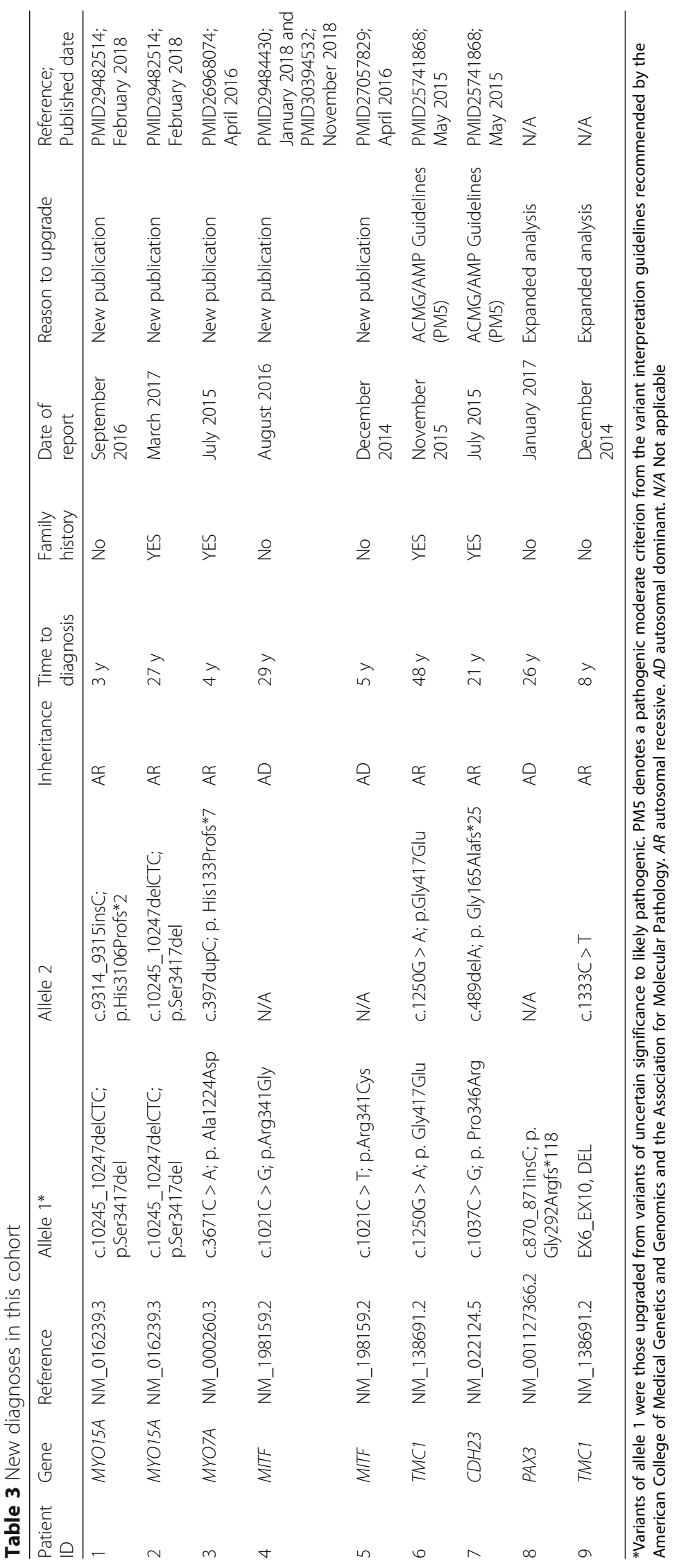


increased by $11 \sim 13 \%$, mainly due to the discovery of new genes linked with the disorders $[9,10]$. By comparison, in our reanalysis new evidence from publications to reclassify the pathogenicity of variants was the leading contributor of novel diagnoses. Furthermore, the dramatic increase in the number of pathogenic variants curated (9210 pathogenic variants per year) [7] reinforces the value of variant reinterpretation for such data.

Although a recent study revealed that reinterpretation of genomic test results should be performed at least every two years [30], and it is clear that periodic reanalysis of undiagnosed cases is beneficial because of the growth in knowledge linking variants and diseases, frequent reassessment is both expensive and time-consuming, rendering this approach to be static in practice. Framework to improve the efficiency of reanalysis is further required.

Consistent with previous publications, GJB2 and $S L C 26 A 4$ were the major genetic causes of hearing loss in the Chinese population; the main contributing variants were GJB2 c.235delC, GJB2 c.109G > A, and SLC26A4 c.919-2A > G [31, 32]. In our reanalysis, we upgraded a three-base deletion, NM_016239.3(MYO15A):c.102 45_10247delCTC, from VUS to pathogenic in light of evidence that it cosegregates with the disease [20]. This variant was detected in two probands (compound heterozygous in patient 1 and homozygous in patient 2). The allele frequency was $0.299 \%$ in our in-house hearing loss patient database, whereas it was $0.06 \%$ in Eastern Asian and was not detected in other ethnic groups in the Genome Aggregation Database [18]. Together, these findings support the notion that this pathogenic allele is enriched in the Asian population.

Many challenges remain for variant interpretation in practice. First, transcript discrepancy can lead to inaccurate variant interpretation [33]. For example, an autosomal dominant variant was curated as NM_198159.2(MITF):c.1021C > G in patient 6, whereas it was reported as NM_000248.3(MITF):c.718C > G [23], creating a significant barrier to discovery for geneticists. Many genes produce multiple transcripts, and determining which should be used as a reference for evaluating the impact of a variant often presents a challenge [34]. Recently, clinically relevant transcripts of deafness-related genes were systematically curated [35], offering a path to unify the use of transcripts in analyzing hereditary hearing loss. The framework for transcript curation and selection might offer a good example of consistency in variant interpretation.

Second, genetic heterogeneity (in terms of alleles and loci) is notable in inherited diseases, especially hearing loss, making it harder to identify causal variants. Allelic heterogeneity sometimes leads to heterogeneity in the clinical phenotype. In our study cohort, patient 8 presented with congenital nonsyndromic hearing loss, leading to the use of HearingCare_81 panel, which mainly targets nonsyndromic hearing loss genes. As a result, the Chinese hotspot variant NM_00400 4.5(GJB2):c.109G > A was detected and reported in a heterozygous state. Our expanded analysis revealed a frameshift autosomal dominant variant, NM_0011 27366.2(PAX3):c.870_871insC, in the original unfiltered files. Since $P A X 3$ is linked with Waardenburg syndrome with variable clinical features [27], it was not included in the HearingCare_81 panel and thus not considered as the molecular etiology of this case. The heterogeneity and penetrance of the phenotype misled audiologists, resulting in this patient initially being undiagnosed.

High levels of locus heterogeneity present another challenge. In the database of Online Mendelian Inheritance in Man, over 100 genes are associated with hearing loss. This means that numerous VUS from different genes were curated and interpreted, presenting a challenge for geneticists in determining which VUS to prioritize and report when no pathogenic variant is detected. To date, professional societies have not provided specific recommendations about VUS reporting [36, 37], and reporting practices for VUS vary dramatically between different laboratories [38].

Third, the interpretation of the criteria in the ACMG/ AMP guidelines is not always consistent between laboratories. For example, the application of variant frequency in publicly available population databases is recommended, whereas the cutoffs are not indicated except for benign variants alone (allele frequency $>5 \%$ ) [17]. Similar ambiguity can be seen in PM3 (For recessive disorders, detected in trans with a pathogenic variant) and PP1 (Cosegregation with disease in multiple affected family members in a gene definitively known to cause the disease), in which stronger conclusions can be drawn on the basis of more data [17]. Recently, expert specifications of the ACMG/AMP variant interpretation guidelines for genes and disorders have been published [39, 40], and the guidelines are expected to continue to become more specific in the future.

Although we did not recontact the patients for the updated results yet, we noted that a number of genetic centers are recontacting patients occasionally or periodically for modified results [41], which may pose ethical and legal issues. For example, reinterpretation may upgrade a participant's results from negative to positive. However, the participant may not want positive genomic results in their medical records for their own reasons. In this circumstance, the participant's autonomy should be respected [42]. Recently, the American Society of Human Genetics developed a position statement to provide necessary guidance, which will facilitate researchers to appropriately operationalize 
patient recontact after reinterpretation of genetic and genomic research results [43].

\section{Conclusions}

In conclusion, this work demonstrates benefits of reanalysis of targeted gene panel data from congenital/prelingual hearing loss patients. A total of 9 previously undiagnosed case obtained diagnosis, improving the overall diagnostic rate from 39 to $43 \%$. New diagnoses are attributed to newly published clinical evidence in the literature, adoption of new interpretation guidelines and expanded analysis range. In spite of the fixed design of targeted gene panels, reanalysis of such data is still beneficial due to the improvements in variant interpretation. We propose that periodical reanalysis should be performed in clinical practice.

\section{Additional files}

Additional file 1: Table S1. Gene list of diagnostic hearing loss panel (HearingCare). (DOCX $42 \mathrm{~kb}$ )

Additional file 2: Table S2. Molecular characteristics of diagnosed patients. (DOCX $26 \mathrm{~kb}$ )

\section{Abbreviations}

ACMG/AMP: American College of Medical Genetics and Genomics and the Association for Molecular Pathology; AD: Autosomal dominant; AR: Autosomal recessive; CNV: Copy number variation; NGS: Next-generation sequencing; VUS: variants of uncertain significance

\section{Acknowledgements}

We express our gratitude to the patients and their families for their kind permission to contribute their data for research use.

\section{Funding}

Funding for this study was provided by National Natural Science Foundation of China (project No. 81572084), and Xiamen Science and Technology Major Project (project No. 3502Z20171006) to Yulin Zhou. The funders of the study had no role in study design, data collection, data analysis, data interpretation, or writing of the manuscript.

\section{Availability of data and materials}

The data used and/or analyzed during the current study are available from the corresponding author on reasonable request. The data are not publicly available due to privacy or ethical restrictions.

\section{Authors' contributions}

JX wrote the first and final draft of the paper with input from YS and YL. YS, JX, SC, JY, JP provided input on data analysis and critical comments on the paper. ZL and LC provided input on the data analysis. JS, Yun Y coordinated the study and commented on the drafts and the final paper. ZP, YZ and SY designed and supervised the conduct of the study, supervised the data analysis, interpreted the data, and reviewed and commented on all drafts and the final paper. ZP and Yaping $Y$ reviewed and commented on all drafts and the final paper. All authors read and approved the final manuscript.

\section{Ethics approval and consent to participate}

The Institutional Review Board of the BGI approved this study (NO. BGI-IRB 18085). All patients, parents or legal guardians signed an informed consent for sample collection.

\section{Consent for publication}

Not applicable.

\section{Competing interests}

$J X, Y L$, JP, ZL, LC, JS, Yun Y and ZP were employed at BGl at the time of submission. Yaping $Y$ is on the Scientific Advisory Board of Veritas Genetics China, and she founded AiLife Diagnostics. No other potential conflict of interest relevant to this article was reported.

\section{Publisher's Note}

Springer Nature remains neutral with regard to jurisdictional claims in published maps and institutional affiliations.

\section{Author details}

${ }^{1}$ Department of Otorhinolaryngology, Union Hospital of Tongji Medical College, Huazhong University of Science and Technology, Wuhan 430022, China. ${ }^{2}$ BGI Genomics, BGI-Shenzhen, Shenzhen 518083, China. ${ }^{3}$ Tianjin Medical Laboratory, BGI-Tianjin, BGI-Shenzhen, Tianjin 300308, China. ${ }^{4}$ Department of Molecular and Human Genetics, Baylor College of Medicine, Houston, TX, USA. ${ }^{5}$ AiLife Diagnostics, 1920 Country Place Pkwy, Pearland, TX 77584, USA. ${ }^{6}$ United Diagnostic and Research Center for Clinical Genetics, School of Public Health of Xiamen University, Xiamen, Fujian 361003, China.

${ }^{7}$ Xiamen Maternal and Child Health Hospital, Xiamen, Fujian 361003, China.

Received: 29 January 2019 Accepted: 14 May 2019

Published online: 28 May 2019

\section{References}

1. Morton CC, Nance WE. Newborn hearing screening--a silent revolution. N Engl J Med. 2006;354(20):2151-64.

2. Ma D, Zhang J, Luo C, et al. Genetic counseling for patients with nonsyndromic hearing impairment directed by gene analysis. Mol Med Rep. 2016;13(3):1967-74.

3. Guan Q, Balciuniene J, Cao K, et al. AUDIOME: a tiered exome sequencingbased comprehensive gene panel for the diagnosis of heterogeneous nonsyndromic sensorineural hearing loss. Genet Med. 2018;20(12):1600-8.

4. Sloan-Heggen $C M$, Bierer $A O$, Shearer $A E$, et al. Comprehensive genetic testing in the clinical evaluation of 1119 patients with hearing loss. Hum Genet. 2016;135(4):441-50.

5. Shearer AE, DeLuca AP, Hildebrand MS, et al. Comprehensive genetic testing for hereditary hearing loss using massively parallel sequencing. Proc Natl Acad Sci U S A. 2010;107(49):21104-9.

6. Sheppard S, Biswas S, Li MH, et al. Utility and limitations of exome sequencing as a genetic diagnostic tool for children with hearing loss. Genet Med. 2018;20(12):1663-76.

7. Wenger AM, Guturu H, Bernstein JA, Bejerano G. Systematic reanalysis of clinical exome data yields additional diagnoses: implications for providers. Genet Med. 2017;19(2):209-14.

8. Gibson KM, Nesbitt A, Cao K et al. Novel findings with reassessment of exome data: implications for validation testing and interpretation of genomic data. Genet Med. 2018;20(3):329-36.

9. Wright CF, McRae JF, Clayton S, et al. Making new genetic diagnoses with old data: iterative reanalysis and reporting from genome-wide data in 1,133 families with developmental disorders. Genet Med. 2018;20(10):1216-23.

10. Ewans $L$, Schofield $D$, Shrestha $R$, et al. Whole-exome sequencing reanalysis at 12 months boosts diagnosis and is cost-effective when applied early in Mendelian disorders. Genet Med. 2018.

11. Li H, Durbin R. Fast and accurate short read alignment with burrowswheeler transform. Bioinformatics. 2009;25(14):1754-60

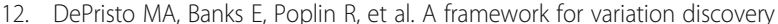
and genotyping using next-generation DNA sequencing data. Nat Genet. 2011:43(5):491-8.

13. Gonzalez-Perez A, Lopez-Bigas N. Improving the assessment of the outcome of nonsynonymous SNVs with a consensus deleteriousness score, Condel. Am J Hum Genet. 2011;88(4):440-9.

14. Zhao S, Xiang J, Fan C, et al. Pilot study of expanded carrier screening for 11 recessive diseases in China: results from 10,476 ethnically diverse couples. Eur J Hum Genet. 2019;27(2):254-62.

15. Richards CS, Bale S, Bellissimo DB, et al. ACMG recommendations for standards for interpretation and reporting of sequence variations: revisions 2007. Genet Med. 2008;10(4):294-300.

16. MacArthur DG, Manolio TA, Dimmock DP, et al. Guidelines for investigating causality of sequence variants in human disease. Nature. 2014:508(7497): $469-76$ 
17. Richards S, Aziz N, Bale S, et al. Standards and guidelines for the interpretation of sequence variants: a joint consensus recommendation of the American College of Medical Genetics and Genomics and the Association for Molecular Pathology. Genet Med. 2015;17(5):405-24.

18. Lek M, Karczewski KJ, Minikel EV, et al. Analysis of protein-coding genetic variation in 60,706 humans. Nature. 2016;536(7616):285-91.

19. Miyagawa M, Nishio SY, Hattori M, et al. Mutations in the MYO15A gene are a significant cause of nonsyndromic hearing loss: massively parallel DNA sequencing-based analysis. Ann Otol Rhinol Laryngol. 2015;124(Suppl 1): 158S-68S.

20. Chang MY, Lee $\mathrm{C}$, Han JH, et al. Expansion of phenotypic spectrum of MYO15A pathogenic variants to include postlingual onset of progressive partial deafness. BMC Med Genet. 2018;19(1):29.

21. Ma Y, Xiao $Y$, Zhang F, et al. Novel compound heterozygous mutations in MYO7A gene associated with autosomal recessive sensorineural hearing loss in a Chinese family. Int J Pediatr Otorhinolaryngol. 2016;83:179-85.

22. Kim SY, Kim AR, Kim NK, et al. Unraveling of enigmatic hearing-impaired GJB2 single heterozygotes by massive parallel sequencing: DFNB1 or not? Medicine (Baltimore). 2016;95(14):e3029.

23. Zhang Z, Chen QD, Zhao LP, et al. A novel variant in MITF in a child from Yunnan-Guizhou plateau with autosomal dominant inheritance of nonsyndromic hearing loss: a case report. Mol Med Rep. 2018;17(4):6054-8.

24. Somashekar PH, Girisha KM, Nampoothiri S, et al. Locus and allelic heterogeneity and phenotypic variability in Waardenburg syndrome. Clin Genet. 2018.

25. Yang T, Kahrizi K, Bazazzadeghan N, Meyer N, Najmabadi H, Smith RJH. A novel mutation adjacent to theBthmouse mutation in theTMC1gene makes this mouse an excellent model of human deafness at the DFNA36 locus. Clin Genet. 2010;77(4):395-8.

26. Mizutari K, Mutai $\mathrm{H}$, Namba K, et al. High prevalence of $\mathrm{CDH} 23$ mutations in patients with congenital high-frequency sporadic or recessively inherited hearing loss. Orphanet J Rare Dis. 2015;10:60.

27. Milunsky JM. Waardenburg Syndrome Type I. GeneReviews. https://www. ncbi.nlm.nih.gov/books/NBK1531/. Accessed on 17 Oct 2018.

28. Lionel AC, Costain G, Monfared N, et al. Improved diagnostic yield compared with targeted gene sequencing panels suggests a role for whole-genome sequencing as a first-tier genetic test. Genet Med. 2018; 20(4):435-43.

29. Gilissen C, Hehir-Kwa JY, Thung DT, et al. Genome sequencing identifies major causes of severe intellectual disability. Nature. 2014:511(7509):344-7.

30. SoRelle JA, Thodeson DM, Arnold S, Gotway G, Park JY. Clinical utility of reinterpreting previously reported genomic epilepsy test results for pediatric patients. JAMA Pediatr. 2018

31. Dai P, Li Q, Huang D, et al. SLC26A4 c.919-2A>G varies among Chinese ethnic groups as a cause of hearing loss. Genet Med. 2008;10(8):586-92.

32. Liu XZ, Xia XJ, Ke XM, et al. The prevalence of connexin 26 ( GJB2) mutations in the Chinese population. Hum Genet. 2002;111(4-5):394-7.

33. Bodian DL, Kothiyal P, Hauser NS. Pitfalls of clinical exome and gene panel testing: alternative transcripts. Genet Med. 2018.

34. den Dunnen JT, Dalgleish R, Maglott DR, et al. HGVS recommendations for the description of sequence variants: 2016 update. Hum Mutat. 2016;37(6): 564-9.

35. DiStefano MT, Hemphill SE, Cushman BJ, et al. Curating clinically relevant transcripts for the interpretation of sequence variants. J Mol Diagn. 2018; 20(6):789-801.

36. Rehm HL, Bale SJ, Bayrak-Toydemir P, et al. ACMG clinical laboratory standards for next-generation sequencing. Genet Med. 2013;15(9):733-47.

37. Matthijs G, Souche E, Alders M, et al. Guidelines for diagnostic nextgeneration sequencing. Eur J Hum Genet. 2016;24(1):2-5.

38. Taber JM, Klein WMP, Lewis KL, Johnston JJ, Biesecker LG, Biesecker BB. Reactions to clinical reinterpretation of a gene variant by participants in a sequencing study. Genet Med. 2018;20(3):337-45.

39. Oza AM, DiStefano MT, Hemphill SE, et al. Expert specification of the ACMG/ AMP variant interpretation guidelines for genetic hearing loss. Hum Mutat. 2018;39(11):1593-613.

40. Kelly MA, Caleshu C, Morales A, et al. Adaptation and validation of the ACMG/AMP variant classification framework for MYH7-associated inherited cardiomyopathies: recommendations by ClinGen's inherited cardiomyopathy expert panel. Genet Med. 2018;20(3):351-9
41. Sirchia F, Carrieri D, Dheensa S, et al. Recontacting or not recontacting? A survey of current practices in clinical genetics centres in Europe. Eur J Hum Genet. 2018;26(7):946-54.

42. Prince AE, Conley JM, Davis AM, Lazaro-Munoz G, Cadigan RJ. Automatic placement of genomic research results in medical records: do researchers have a duty? Should participants have a choice? J Law Med Ethics. 2015; 43(4):827-42.

43. Bombard $Y$, Brothers KB, Fitzgerald-Butt $\mathrm{S}$, et al. The responsibility to Recontact research participants after reinterpretation of genetic and genomic research results. Am J Hum Genet. 2019;104(4):578-95.
Ready to submit your research? Choose BMC and benefit from:

- fast, convenient online submission

- thorough peer review by experienced researchers in your field

- rapid publication on acceptance

- support for research data, including large and complex data types

- gold Open Access which fosters wider collaboration and increased citations

- maximum visibility for your research: over $100 \mathrm{M}$ website views per year

At BMC, research is always in progress.

Learn more biomedcentral.com/submissions 